\title{
New molecular targets against cervical cancer
}

\author{
This article was published in the following Dove Press journal: \\ International Journal of Women's Health \\ 5 December 2014 \\ Number of times this article has been viewed
}

\author{
Alfonso Duenas-Gonzalez ${ }^{1,2}$ \\ Alberto Serrano-Olvera ${ }^{3}$ \\ Lucely Cetina ${ }^{4}$ \\ Jaime Coronel ${ }^{4}$ \\ 'Unit of Biomedical Research in \\ Cancer, Instituto de Investigaciones \\ Biomedicas UNAM/Instituto Nacional \\ de Cancerologia, Mexico City, \\ ${ }^{2}$ ISSEMyM Cancer Center, Toluca, \\ ${ }^{3}$ Medical Oncology Service, ABC \\ Medical Center, Mexico City, ${ }^{4}$ Division \\ of Clinical Research, Instituto \\ Nacional de Cancerologia, Mexico \\ City, Mexico \\ On behalf of the Tumor Study Group
}

Correspondence: Alfonso DuenasGonzalez

Unidad de Investigacion Biomedica en Cancer, San Fernando 22, Tlalpan I4080, Mexico City, Mexico

Email alfonso_duenasg@yahoo.com

\begin{abstract}
Cervical cancer is the third most commonly diagnosed cancer worldwide and the fourth leading cause of cancer death in women. Major advances but still insufficient achievements in the treatment of locally advanced and high-risk early stage patients have occurred in the last decade with the incorporation of concurrent cisplatin with radiation and, lately, gemcitabine added to cisplatin chemoradiation. Despite a number of clinical studies incorporating moleculartargeted therapy as radiosensitizers being in progress, so far, only antiangiogenic therapy with bevacizumab added to cisplatin chemoradiation has demonstrated safety and shown encouraging results in a Phase II study. In advanced disease, cisplatin doublets do not have a great impact on the natural history of the disease with median survival rates not exceeding 13 months. The first Phase III study of bevacizumab, added to cisplatin or a non-cisplatin-containing doublet, showed significant increase in both overall survival and progression-free survival. Further studies are needed before bevacizumab plus chemotherapy can be considered the standard of care for advanced disease. Characterization of the mutational landscape of cervical cancer has already been initiated, indicating that, for now, few of these targetable alterations match with available agents. Progress in both the mutational landscape knowledge and developments of novel targeted therapies may result in more effective and individualized treatments for cervical cancer. The potential efficacy of knocking down the key alterations in cervical cancer - E6 and E7 human papillomavirus oncoproteins - must not be overlooked.
\end{abstract}

Keywords: cervical cancer, molecular-targeted therapy, bevacizumab, HPV

\section{Introduction}

Cervical cancer is a malignant disease that can be prevented; however, it is the third most commonly diagnosed cancer worldwide and the fourth leading cause of cancer death in women, accounting for $9 \%(529,800)$ of total new cases of cancer and $8 \%$ $(275,100)$ of all cancer deaths. This cancer mainly affects socially disadvantaged women; therefore, $83 \%$ of cases occur in developing countries, while in developed countries, cervical cancer accounts for only $3.6 \%$ of new cancer cases. ${ }^{1}$

Cervical cancer is clinically staged according to International Federation of Gynecology and Obstetrics (FIGO) guidelines, though regarding treatment it can be grouped into three subgroups: the earliest stages IA (microinvasive diseases) to IIA $_{1}$ (tumors affecting the upper third of the vagina measuring $<4 \mathrm{~cm}$, which are usually treated by surgical procedures as definitive treatment). Regarding surgical treatment for early stages, conization with adequate excision margins instead of simple hysterectomy is considered effective for microinvasive carcinoma (stage $\mathrm{IA}_{1}$ ) and can preserve fertility; however, evidence for its benefit is from observational studies only. 
No randomized clinical trials exist to assess the best treatment for IA $_{2}$ disease but acceptable approaches include conization or simple hysterectomy with pelvic lymphadenectomy and radical hysterectomy as well. For small volume macroscopic disease (IB ${ }_{1}$ and IIA $A_{1}$, radical hysterectomy continues to be the standard of care but in this setting radical trachelectomy plus lymphadenectomy can be used to preserve fertility. ${ }^{2}$ Neoadjuvant chemotherapy followed by surgery in the early stages of disease has not shown benefit in survival parameters but is commonly used to downstage disease when fertilitypreserving procedures are done. ${ }^{3}$ Early-stage patients do not require adjuvant systemic treatment unless there are high-risk factors for recurrence in the surgical specimen. These cases are usually prescribed with adjuvant chemoradiation. ${ }^{4,5}$

Intermediate stages, which are the locally advanced cases (IB ${ }_{2}-$ IVA), require primary chemoradiation as definitive treatment. For this subgroup of patients, adjuvant systemic chemotherapy and neoadjuvant chemoradiation are modalities under study in randomized Phase III trials. ${ }^{6,7}$ Finally, systemic chemotherapy is the treatment of choice for most advanced cases that comprise IVB stages as well as patients with recurrent or persistent disease not amenable to curative treatment. ${ }^{8}$ A recent Phase III study has demonstrated that adding bevacizumab increases progression-free survival (PFS) and overall survival (OS). ${ }^{9}$

\section{Current standard of treatment for locally advanced/early-stage high-risk patients}

The addition of chemotherapy to radiation, also known as chemoradiation, improves OS (hazard ratio [HR] 0.71; $P<0.0001$ ) whether platinum is used (HR 0.70; $P<0.0001$ ) or not (HR $0.81 ; P=0.20$ ). PFS is improved as well (HR 0.61 ; $P<0.0001)$. Thus, the absolute benefits in PFS and OS are $16 \%$ (95\% confidence interval [CI] 13\%-19\%) and 12\% (95\% CI 8\%-16\%), respectively. Significant benefits of chemoradiation on both local (HR 0.61; $P<0.0001)$ and distant recurrence (HR $0.57 ; P<0.0001$ ) are also observed. This overall improved efficacy is at the expense of increased but manageable grade $3 / 4$ hematological and gastrointestinal toxicities. ${ }^{10}$ These regimens - commonly known as concurrent chemoradiation (most commonly using cisplatin at $40 \mathrm{mg} / \mathrm{m}^{2}$ weekly for 6 weeks) - yield 5-year survival rates ranging from $60 \%-70 \%$, and are the current standard of care. A recent meta-analysis and systematic review compared outcomes and disease control rates of locally advanced disease treated with single agent weekly cisplatin chemoradiation against chemoradiation with a cisplatin combination regimen.
The results show that patients treated with the more intensive regimens experienced significant improvements in survival and locoregional control. In particular, the risk of death reduced by $35 \%$, and the risk of progression reduced by $29 \%$. Though toxicities were largely underreported in the analyzed publications, thrombocytopenia, neutropenia, and acute gastrointestinal toxicities were statistically higher in the cisplatin combination arms. ${ }^{11}$ These results clearly point to the fact that chemoradiation is yet to reach a ceiling in efficacy; hence, new drug combinations and radiation should be explored to fully exploit the efficacy of this modality.

\section{Current standard of treatment for advanced disease}

A Gynecologic Oncology Group study - GOG-204 - compared four cisplatin doublets (paclitaxel cisplatin reference arm) against vinorelbine cisplatin, gemcitabine cisplatin, and topotecan cisplatin. Treatment schedules were the following: cisplatin at $50 \mathrm{mg} / \mathrm{m}^{2}$ in each of the arms; paclitaxel at $135 \mathrm{mg} / \mathrm{m}^{2}$ over 24 hours plus cisplatin $50 \mathrm{mg} / \mathrm{m}^{2} /$ day; vinorelbine $30 \mathrm{mg} / \mathrm{m}^{2}$ on Day 1 and Day 8; gemcitabine $1,000 \mathrm{mg} / \mathrm{m}^{2}$ on Day 1 and Day 8 , and topotecan $0.75 \mathrm{mg} / \mathrm{m}^{2}$ on Days 1-3. A total of 513 patients were enrolled when a planned interim analysis recommended early closure due to futility. Experimental-to-paclitaxel cisplatin HRs of death were as follows: 1.15 (95\% CI 0.79-1.67) for vinorelbine cisplatin, 1.32 (95\% CI 0.91-1.92) for gemcitabine cisplatin, and 1.26 (95\% CI 0.86-1.82) for topotecan cisplatin. HRs for PFS were 1.36 (95\% CI 0.97-1.90) for vinorelbine cisplatin, 1.39 (95\% CI 0.99-1.96) for gemcitabine cisplatin, and 1.27 (95\% CI 0.90-1.78) for topotecan cisplatin. There were no significant differences in regard to response rate, which were $29.5 \%, 25.9 \%, 22.3 \%$, and $23.4 \%$ for the cisplatin combinations with paclitaxel, vinorelbine, gemcitabine, and topotecan, respectively. Likewise, quality of life showed no differences among the groups. ${ }^{12,13}$

After the results of the GOG-204 study demonstrating nonsuperiority of any of these four regimens, GOG-240 incorporating bevacizumab to chemotherapy was undertaken and recently the results were reported. ${ }^{9}$ This study randomized 452 advanced cervical cancer patients - using a $2 \times 2$ factorial design - to chemotherapy with or without bevacizumab at a dose of $15 \mathrm{mg} / \mathrm{kg}$. Chemotherapy consisted of cisplatin at a dose of $50 \mathrm{mg} / \mathrm{m}^{2}$ plus paclitaxel at 135 or $175 \mathrm{mg} / \mathrm{m}^{2}$ or topotecan at $0.75 \mathrm{mg} / \mathrm{m}^{2}$ on Days $1-3$, plus paclitaxel at a dose of $175 \mathrm{mg} / \mathrm{m}^{2}$ on Day 1 . Cycles were repeated every 21 days until disease progression. Groups were well balanced for main clinicopathological factors, including 
previous use of radiosensitizers. Results demonstrated that topotecan-paclitaxel was not superior to cisplatin-paclitaxel (HR for death 1.20) and with data for the two chemotherapy regimens combined, the addition of bevacizumab to chemotherapy was associated with increased OS (17.0 versus 13.3 months; HR $0.71 ; 98 \%$ CI $0.54-0.95 ; P=0.004$ in a one-sided test). A significant improvement in PFS was also seen (8.2 versus 5.9 months; HR 0.67; 95\% CI 0.54-0.82). The treatment was in general well tolerated and no unexpected adverse events resulting from bevacizumab were observed. In bevacizumab-treated patients, there were more grade 4 neutropenia events ( $26 \%$ versus $35 \%$ ), grade $\geq 3$ thromboembolisms ( $1 \%$ versus $8 \%$ ), and grade $>2$ hypertension. Gastrointestinal fistula, gastrointestinal perforation, and genitourinary fistula (all grade $\geq 3$ ) were only observed in patients treated with bevacizumab $(3 \%, 2 \%$, and $2 \%$, respectively). Analysis of quality of life using the Functional Assessment of Cancer Therapy - Trial Outcome Index (FACT-CX TOI) score showed a modest, statistically nonsignificant decline in patients receiving the monoclonal antibody. ${ }^{9}$ These data led the authors to conclude that bevacizumab is the first targeted therapy shown to increase survival parameters in advanced cervical cancer without decreasing quality of life.

\section{Molecular-targeted therapy in cervical cancer}

Post-genomic drugs (referred to as targeted agents) in contraposition to pre-genomic drugs (classical cytotoxic drugs) were intended to have a clinical development based primarily on whether or not the tumor has the molecular alteration to be targeted. The paradigmatic case of imatinib in chronic myelogenous leukemia that transformed the disease - changing the median OS from 4 years to an estimated 19-25 years - has not been mirrored so far in solid tumors. However, the current understanding of how the molecular circuitry is altered in cancer and how it should be modulated to obtain a therapeutic gain is far from being completed; hence, in many cases, the clinical results of early-phase studies are the stronger indicators of whether or not the pathway targeted with a particular agent is of clinical relevance. In addition, predictive factors of response to these therapies are available in a few cases only. These facts have led to a clinical drug development quite similar to that employed for cytotoxic chemotherapy, ie, the testing of molecular-targeted drugs against every tumor type hoping that the targeted molecule or pathway could be relevant and translate into clinical efficacy.

No targeted drug has been developed specifically for cervical cancer. Beyond the fact that the current model of cancer drug development is market driven and locally advanced and advanced cervical cancer are not attractive ones, data on targetable genetic alterations in cervical cancer are far behind the knowledge for other tumor types. In this sense, until recently, information from the Sanger database was the only one available regarding mutation frequency of potentially targetable alterations in cervical cancer. ${ }^{14}$ Recently, a comprehensive genetic landscape analysis for cervical cancer was published. In the study, ${ }^{15}$ whole-exome sequencing analysis of 115 cervical carcinoma-normal paired samples, transcriptome sequencing of 79 cases, and whole-genome sequencing of 14 tumor-normal pairs were sequenced. As expected, mutations at PIK3CA, PTEN, and STK11 were present in $14 \%, 6 \%$, and $4 \%$, respectively. Among previously unknown somatic mutations, authors found $\mathrm{E} 322 \mathrm{~K}$ substitutions in the $M A P K 1$ gene $(8 \%)$, inactivating mutations in the $H L A-B$ gene ( $9 \%$ ), and mutations in EP300 (16\%), FBXW7 (15\%), NFE2L2 (4\%), TP53 (5\%), and ERBB2 (6\%). Somatic ELF3 (13\%) and $C B F B(8 \%)$ mutations in 24 adenocarcinomas were also observed. Interestingly, three out of the six ERBB2 mutations (S310F, S310Y, and V842I) are known oncogenic driver mutations.

\section{Current results of molecular- targeted drugs in cervical cancer}

As discussed above in regard to the rationale used for testing molecular-targeted agents in cervical cancer, it is not surprising that results in general are poor with the exception of bevacizumab.

\section{EGFR antagonists}

The availability of agents against EGFR, either monoclonal antibodies or small molecule inhibitors, and their success in lung and colorectal cancer among others, prompted evaluation of these agents in cervical cancer. None of these agents have progressed to Phase III trials yet, but so far they have not shown impressive results in their early testing either as single agents or in combination with chemotherapy or radiation.

Erlotinib has been shown to not have clinical activity as a monotherapy in advanced disease, ${ }^{16}$ though it was safely administered along with cisplatin chemoradiation in a Phase I study. ${ }^{17}$ The same group recently reported the results of a Phase II study in 36 patients concluding that this regimen of erlotinib with chemoradiation is promising with an OS and PFS of $80.6 \%$ and $73.8 \%$, respectively. ${ }^{18}$ Gefitinib has also been evaluated in advanced disease reporting minimal activity in one patient, ${ }^{19}$ whereas a second study in Asian patients 
reported one complete response, one partial response, and four stable disease in a cohort of 20 patients. ${ }^{20}$ The results with cetuximab are no better. As a single agent in advanced disease it has minimal or no activity; ${ }^{21,22}$ however, in combination with cisplatin, although tolerated, it does not appear to increase the efficacy of single agent cisplatin. ${ }^{23}$ On the contrary, when used with a combination of cisplatin and topotecan, the triple combination induced a high rate of adverse/fatal events. ${ }^{24}$ Cetuximab has also been evaluated in combination with radiation and cisplatin. A Phase I study ${ }^{25}$ showed that the combination is feasible only with pelvic radiation but not with extended-field radiation. No results are yet published from this combination in Phase II studies.

Panitumumab and nimotuzumab - two other anti-EGFR antibodies - are being evaluated in cervical cancer. The results of these trials are awaited; however, to date, the evidence indicates that the results of targeted therapy based on EGFR blocking are behind those obtained in head and neck and colorectal cancer with anti-EGFR antibodies and a subset of non-small-cell lung cancer with tyrosine kinase inhibitors gefitinib and erlotinib. Taken together, these data may suggest that growth regulation by the EGFR pathway in cervical cancer is not the dominant oncogenic driver. Supporting this view, in a model of cervical cancer cell line C41 transfected with a dexamethasone-induced promoter for human papillomavirus (HPV) 18 E6/E7 genes, the downregulation or upregulation of these viral oncogenes did not lead to changes in the expression of EGFR under identical culture conditions and, interestingly, the growth rate of the cells correlated with the level of viral gene products rather than with the expression of the EGFR. ${ }^{26}$

\section{Multitargeted tyrosine kinase inhibitors}

Among this miscellaneous group of agents, imatinib (BCR-ABL, c-KIT, PDGFR) and sunitinib (PDGFR $\alpha$, PDGFR $\beta$, VEGFR1, VEGFR2, c-kit, and FLT3) have been evaluated in the advanced disease setting. No evidence of response nor suggestion of increased stabilization of disease were observed. ${ }^{27,28}$ No results have yet been published on other multitargeted tyrosine kinase inhibitors such as sorafenib.

\section{Miscellaneous targeted drugs}

A number of other targeted drugs are being tested in cervical cancer either alone or in combination with chemotherapy or chemoradiation. Among these is the mTOR inhibitor temsirolimus, which showed modest activity as a single agent in advanced disease, ${ }^{29}$ however, in combination with topotecan, it was not tolerated in patients with previous pelvic radiation. ${ }^{30}$ Celecoxib - a cyclooxygenase- 2 inhibitor - was added to cisplatin 5-fluorouracil chemoradiation in locally advanced disease with no impressive results; ${ }^{31}$ hence, no clinical trials with this agent are currently ongoing in cervical cancer. Because HPV E6 targets p53 for degradation through the ubiquitin-proteasome system, proteasome inhibitors are good candidates to increase wild-type p53 expression in cervical carcinoma cells. A number of proteasome inhibitors, including MG132 and bortezomib, have shown their ability to increase p53 protein levels, to induce apoptosis, and to reduce VEGF, as well as to increase radiosensitization in cervical cancer cells. ${ }^{32}$ Two terminated ClinicalTrials.gov studies assessed bortezomib with irinotecan (NCT00106262) and bortezomib plus chemoradiation (NCT00329589) in advanced and locally advanced cervical cancer, but no results have yet been published. Preclinical data suggest that poly (adenosine diphosphate ribose) polymerase inhibitors may potentiate the effects of radiation in several tumor types and induce apoptosis in cervical cancer cells. ${ }^{33,34}$ A Phase I/II study of veliparib associated with paclitaxel (NCT01281852) as well as a Phase II study of veliparib with topotecan plus G-CSF/pegylated G-CSF (NCT01266447) are ongoing for the treatment of advanced cervical cancer. Likewise, olaparib plus paclitaxel is being tested in advanced gynecological cancers (NCT01237067).

Epigenetic therapy of cancer is a promising modality under development, particularly with the use of DNA methyltransferase and histone deacetylase inhibitors either alone or in combination. It is known that HPV E6/E7 oncoproteins interact with DNA methyltransferase and histone deacetylase enzymes, which - in addition to aiding in cell transformation - may also participate in the transcriptional inactivation of a high number of tumor suppressor genes. ${ }^{35}$ When hydralazine - an antihypertensive agent - and the antiepileptic magnesium valproate are repositioned as DNA methyltransferase and histone deacetylase inhibitors, respectively, they show radiosensitization in cervical cancer cells ${ }^{36}$ and are highly effective when added to chemoradiation with cisplatin for locally advanced disease. ${ }^{37}$ Preliminary results of a randomized Phase III trial comparing hydralazine-magnesium valproate against placebo in advanced cervical cancer patients receiving standard cisplatin topotecan showed - at a median follow-up time of 7 months (range 1-22 months) - a median PFS of 10 versus 6 months 
$(P=0.0384)$ in favor of the experimental arm, suggesting that epigenetic therapy could be effective in cervical cancer. ${ }^{38}$

\section{Antiangiogenesis agents}

As early as 1975, the importance of angiogenesis for cervical cancer was recognized. ${ }^{39}$ VEGF is one of the most important factors involved in regulating angiogenesis, and several studies have demonstrated that its expression correlates with the more aggressive clinical-pathological characteristics of the disease and with prognosis. ${ }^{40-44}$ The increased production of VEGF within cervical neoplasms led to the testing of the monoclonal antibody bevacizumab in this tumor. Among early studies with bevacizumab, six women with metastatic disease who previously received 5 -fluorouracil and then bevacizumab had a $67 \%$ clinical benefit rate with median PFS of 4.3 months,${ }^{45}$ whereas Tan et al reported the case of a patient with a recurrent tumor who had disease improvement with the use of bevacizumab and carboplatin. ${ }^{46} \mathrm{~A}$ Phase II trial to assess the efficacy and tolerability of bevacizumab was undertaken by the Gynecologic Oncology Group. Bevacizumab was administered at $15 \mathrm{mg} / \mathrm{kg}$ intravenously every 3 weeks until disease progression or prohibitive toxicity. Five (10.9\%) partial responses and eleven (23.9\%) stable disease were registered among the 46 patients studied, which translated into a median PFS and OS of 3.4 and 7.9 months, respectively. The treatment was well tolerated; there were eight events of grade 3 hematologic toxicity, five patients had deep vein thrombosis, one had grade 4 vaginal bleeding, and one had grade 4 urinary fistula. Other grade 3 events included high blood pressure, but none required discontinuation of therapy. The activity of bevacizumab observed in this study was remarkable, and was superior to the activity observed for any other single agent studied by the Gynecologic Oncology Group in the setting of treatment failure with one prior regimen for advanced cervical cancer. ${ }^{47}$ These results and those from GOG-204 led to the GOG-240 study discussed above, which is touted as the new standard of treatment for advanced disease. Whether or not the bevacizumab-containing regimen will be widely accepted remains to be demonstrated. Almost at the same time that results of GOG-240 were released in the American Society of Clinical Oncology's 2013 meeting, the results of a small Phase II study in a similar patient population were published. ${ }^{48}$ In this multicentric study, 27 patients received the same regimen of bevacizumab with standard cisplatin-topotecan. Compared with the GOG240 study, the response rate observed was $45 \%$ (one complete response $[4 \% ; 80 \%$ CI $0.4 \%-14 \%$ ] and eight partial responses [31\%; 80\% CI 19\%-45\%]). At a median follow-up of 10 months (range 1.7-33.4 months), median PFS was 7.1 months ( $80 \%$ CI $4.7-10.1$ months) and median OS was 13.2 months ( $80 \%$ CI $8.0-15.4$ months). Of concern, the regimen resulted in excessive toxicity, with nearly $80 \%$ of patients requiring unanticipated hospital admissions to manage adverse events and/or to provide supportive care. In addition, one death possibly related to therapy and a high rate of severe constitutional toxicities led to approximately $19 \%$ of patients discontinuing protocol treatment. The results obtained in this study are very similar to the results in the control arm of GOG-240. Whether these poor results were due to the regimen of cisplatin-topotecan or due to other reasons originated from the study population are not known; nevertheless, these results clearly suggest that additional studies are needed before it can be accepted as the standard of care. Another unresolved issue for the regimen is its applicability to patients with stage IVB disease, as this subgroup of advanced disease represented only $16.8 \%$ of the treated population in GOG- $240^{9}$ and less than $10 \%$ in the Phase II study discussed above. ${ }^{48}$ Cost-effectiveness analyses are also warranted because of the societal burden involved in making expensive therapies available to those in greatest need.

As has occurred with the clinical development of bevacizumab for other tumors, it has also been tested in locally advanced disease as primary treatment added to standard cisplatin chemoradiation. A Radiation Therapy Oncology Group study - RTOG 0417 - demonstrates the feasibility, safety, and encouraging oncologic outcomes with the addition of bevacizumab to standard chemoradiotherapy. In this Phase II multicentric study, 49 bulky stage IB-IIIB patients from 28 institutions were enrolled from 2006-2009. Patients received standard weekly cisplatin at $40 \mathrm{mg} / \mathrm{m}^{2}$ and pelvic radiation plus brachytherapy. Bevacizumab was administered at 10 $\mathrm{mg} / \mathrm{kg}$ intravenously every 2 weeks for three cycles during chemoradiation. There was good compliance with the treatment and it resulted in minimal toxicity. The most common was hematologic, grade 3 toxicity in 13 patients $(26.5 \%)$ and grade 4 toxicity in five patients (10.2\%). There were no grade 4 gastrointestinal toxicities nor treatment-related deaths. The 3 -year OS and disease-free survival were $81.3 \%$ (95\% CI $67.2 \%-89.8 \%$ ) and $68.7 \%$ (95\% CI 53.5\%-79.8\%), respectively. ${ }^{49}$ These results compare favorably to those achieved with cisplatin chemoradiation and, interestingly, are similar to those reported in the experimental arm of the cisplatingemcitabine chemoradiation trial that compared this regimen against standard cisplatin chemoradiation. ${ }^{50}$ These results 
clearly support further testing of this regimen. Continuing bevacizumab after chemoradiation is of particular interest.

Other antiangiogenic agents are being evaluated in advanced cervical cancer. The activity of pazopanib was compared against lapatinib (an HER1/HER2 tyrosine kinase inhibitor) and against both in 230 patients with pretreated advanced cervical cancer. The combination was discontinued and the final analysis was done only in those patients treated with either lapatinib or pazopanib. Pazopanib was well tolerated and resulted in improved PFS - the endpoint of the study (HR 0.66; 90\% CI 0.48-0.91; $P=0.013$ ) and OS (HR 0.67; 90\% CI 0.46-0.99; $P=0.045$ ). Response rates were $9 \%$ and $5 \%$ for pazopanib and lapatinib, respectively. ${ }^{51}$ Although this study was not powered for OS, an updated publication on this trial reported a median OS of 44.1 weeks for lapatinib and one of 49.7 weeks for patients who received pazopanib (HR 0.96; 90\% CI 0.71-1.30; $P=0.407$ ). ${ }^{52}$ Combining pazopanib with chemotherapy is also being explored. A Phase I/II study (NCT00561795) has been completed in which newly diagnosed advanced gynecological malignancies, including the cervix, received carboplatin (at two dose levels) plus paclitaxel and pazopanib. This trial is yet to be reported. No Phase III trials are registered with this agent. Cediranib - another orally bioavailable small molecule inhibitor of VEGFR-1, -2, and $-3^{53}$ - is under evaluation in a randomized Phase II trial comparing carboplatin paclitaxel with or without cediranib in advanced cervical cancer (NCT01229930). Brivanib - a dual tyrosine kinase inhibitor that shows selectivity against VEFGR-2 and FGFR ${ }^{54}$ - is another small molecule angiogenesis inhibitor that is being tested as single agent in a Phase II study for patients with advanced cervical cancer (NCT01267253).

\section{HPV-directed therapy}

It is remarkable that drug development for cervical cancer has not placed a major effort in targeting the key molecular alteration that leads to cervical cancer development. HPV has long been established as the etiology of cervical cancer (and other neoplasias) by the regular presence of HPV DNA and its E6/E7 viral oncogene expression. ${ }^{55}$ Of utmost importance is the solid evidence that cervical cancer cells require the expression of these viral oncogenes for maintaining the malignant phenotype via interaction of viral oncoproteins with growthregulating host cell proteins; ${ }^{.56}$ thus, essentially HPV oncoproteins directly or indirectly exert oncogenic effects upon cancer hallmarks by: 1) resisting cell death, 2) inducing angiogenesis, 3) sustaining proliferative signaling, 4) evading growth suppressors, 5) activating invasion and metastasis, and 6) enabling replicative immortality. The most compelling evidence on the key role of HPV oncoproteins in cervical cancer is the demonstration that repressing endogenous HPV oncogenes E6/E7 mobilizes the p53 and Rb pathways in an orderly fashion to deliver growth inhibitory signals to the cells. ${ }^{59}$ Therefore, unlike most cancers in which the brakes on cell growth are broken, in HeLa cells - which are reminiscent of an advanced cervical cancer ${ }^{60}$ - the driver is asleep; hence, downregulation of E6/E7 is sufficient to wake up the driver and impose growth control. ${ }^{61}$ The antitumor effects of knocking down the function of E6/E7 oncoproteins has been widely demonstrated by a variety of approaches. ${ }^{62-68}$ Since the first small inhibitory RNA (siRNA)-based RNA interference agent entered clinical trial in 2004, there have been more than 20 siRNA-based RNA interference therapeutics entering clinical trial for local or systemic disease treatment and the number is growing thanks to the development of better siRNA delivery vehicles that hopefully may increase their therapeutic potency and reduce off-target effects. ${ }^{69}$ This is clearly a promising research avenue to be explored for cervical cancer treatment.

siRNA-based RNA interference is not the only potential manner of HPV-based cervical cancer treatment. Small molecules, including antivirals, are also another potential way of blocking either directly or indirectly the oncogenic actions of E6/E7. Small molecule RITA blocks p53 ubiquitination by preventing p53 interaction with E6AP, which is required for the HPV E6-mediated degradation that leads to substantial suppression of cervical cancer xenografts in vivo. ${ }^{70}$ Withaferin $\mathrm{A}$ - the active component of the medicinal plant Withania somnifera - is able to downregulate the expression of HPV E6 and E7 oncoproteins and to induce accumulation of p53 and increase levels of p21, causing cell cycle arrest and strong antitumoral effect in nude mice xenografts. ${ }^{71}$ Lignans from the creosote bush can repress the HPV-E6 gene promoter leading to at least five-fold less E6 protein expression, resulting in a stabilized and transcriptionally active p53 protein within treated HPV-containing tumor cells. ${ }^{72}$ In this respect, several platforms for screening compounds capable of disrupting the interaction of E6 with E6AP to interfere with the ability of E6 to promote p53 degradation, are promising for finding specific drugs for HPV-related tumors. ${ }^{73-75}$

Broad-spectrum antivirals against DNA viruses are also potentially effective in HPV-related diseases. Cidofovir is a US Food and Drug Administration-approved antiviral against cytomegalovirus retinitis. Cidofovir reduces E6 and E7 expression in cervical and other HPV-associated carcinomas at the transcriptional level, which leads to an 
accumulation of active p53 and $\mathrm{Rb}$ associated with the induction of cyclin-dependent kinase inhibitor p21. Its antitumor activity has been shown in vitro and in vivo against HPV-associated carcinomas and it is also an effective radiosensitizer in HPV-positive - but not HPVnegative - cells. ${ }^{76-78}$ This agent has been used in patients with either laryngeal, esophageal/pharyngeal, or genital HPV-induced proliferative lesions with promising results. ${ }^{79}$ In cervical preinvasive disease (biopsy-proven cervical intraepithelial neoplasia 2+), a double-blind randomized Phase II study of topical cidofovir or placebo that allocated 23 patients to cidofovir and 25 to placebo demonstrated that viral clearance was statistically higher in the active group than in the placebo group $(60.8 \%$ versus $20 \% ; P<0.01)$, suggesting its potential use for this condition. ${ }^{80}$ Despite the strong rationale of using anti-HPV-targeted agents, the clinical development of these agents is quite limited. No clinical trials using a nucleic acid-based strategy against cervical cancer have yet been reported. A clinical trial of cidofovir as a radiosensitizer added to carboplatin and radiation is ongoing (NCT00811408).

\section{Conclusion}

Globally, cervical cancer remains an important threat for women's health, particularly for those living in developing regions of the world. Major advances, but still insufficient achievements, in the treatment of locally advanced and highrisk early stage patients have occurred in the last decade with the incorporation of concurrent cisplatin with radiation and, lately, gemcitabine added to cisplatin chemoradiation. Although a number of clinical studies incorporating molecular-targeted therapy as radiosensitizers are in progress, so far, only antiangiogenic therapy with bevacizumab added to cisplatin chemoradiation has demonstrated safety and shown encouraging results in a Phase II study. ${ }^{49}$

In the setting of advanced/recurrent/metastatic disease, while statistically significant better survival rates are achieved with cisplatin doublets against cisplatin alone, they do not have a great impact on the natural history of the disease as median survival does not exceed 13 months with either of these doublets and 3-year survival is below 20\%. The first Phase III study of bevacizumab - the first targeted drug evaluated in a Phase III trial - showed that when added to cisplatin or a non-cisplatin-containing doublet, there was a significant increase in both OS and PFS. Thus, median survival increased from 13.3 months to 17 months, but still without major impact on the 3-year survival rate. Further studies are needed before bevacizumab plus chemotherapy can be considered the standard of care for advanced disease. On the other hand, characterization of the mutational landscape of cervical cancer has already been initiated, indicating that, for now, few of these targetable alterations match with available agents. Progress in both the mutational landscape knowledge and developments of novel targeted therapies may result in more effective and individualized treatments for cervical cancer. The potential therapeutic efficacy of knocking down the key alterations in cervical cancer - E6 and E7 HPV oncoproteins - must not be overlooked.

\section{Disclosure}

The authors report no conflicts of interest in this work.

\section{References}

1. Jemal A, Bray F, Center MM, Ferlay J, Ward E, Forman D. Global cancer statistics. CA Cancer J Clin. 2011;61(2):69-90.

2. Martin-Hirsch PL, Wood NJ. Cervical cancer. Clin Evid (Online). 2011;2011:0818.

3. Plante M. Evolution in fertility-preserving options for early-stage cervical cancer: radical trachelectomy, simple trachelectomy, neoadjuvant chemotherapy. Int J Gynecol Cancer. 2013;23(6):982-989.

4. Eifel PJ, Winter K, Morris M, et al. Pelvic irradiation with concurrent chemotherapy versus pelvic and para-aortic irradiation for high-risk cervical cancer: an update of Radiation Therapy Oncology Group trial (RTOG) 90-01. J Clin Oncol. 2004;22(5):872-880.

5. Peters WA 3rd, Liu PY, Barrett RJ 2nd, et al. Concurrent chemotherapy and pelvic radiation therapy compared with pelvic radiation therapy alone as adjuvant therapy after radical surgery in high-risk early-stage cancer of the cervix. J Clin Oncol. 2000;18(8):1606-1613.

6. Gynecologic Oncology Group. Cisplatin and radiation therapy with or without carboplatin and paclitaxel in patients with locally advanced cervical cancer. Available from: http://clinicaltrials.gov/show/NCT01414608. NLM identifier: NCT01414608. Accessed June 15, 2014.

7. University College, London. Induction chemotherapy plus chemoradiation as first line treatment for locally advanced cervical cancer (INTERLACE). Available from: http://clinicaltrials.gov/show/NCT01566240. NLM identifier: NCT01566240. Accessed June 15, 2014.

8. Scatchard K, Forrest JL, Flubacher M, Cornes P, Williams C. Chemotherapy for metastatic and recurrent cervical cancer [review]. Cochrane Database Syst Rev. 2012;10:CD006469.

9. Tewari KS, Sill MW, Long HJ 3rd, et al. Improved survival with bevacizumab in advanced cervical cancer. $N$ Engl J Med. 2014;370(8): 734-743.

10. Chemoradiotherapy for Cervical Cancer Meta-Analysis Collaboration. Reducing uncertainties about the effects of chemoradiotherapy for cervical cancer: a systematic review and meta-analysis of individual patient data from 18 randomized trials. J Clin Oncol. 2008;26(35): 5802-5812.

11. Petrelli F, De Stefani A, Raspagliesi F, Lorusso D, Barni S. Radiotherapy with concurrent cisplatin-based doublet or weekly cisplatin for cervical cancer: a systematic review and meta-analysis. Gynecol Oncol. 2014;134(1):166-171.

12. Monk BJ, Sill MW, McMeekin DS, et al. Phase III trial of four cisplatincontaining doublet combinations in stage IVB, recurrent, or persistent cervical carcinoma: a Gynecologic Oncology Group study. J Clin Oncol. 2009;27(28):4649-4655.

13. Cella D, Huang HQ, Monk BJ, et al. Health-related quality of life outcomes associated with four cisplatin-based doublet chemotherapy regimens for stage IVB recurrent or persistent cervical cancer: a Gynecologic Oncology Group study. Gynecol Oncol. 2010;119(3):531-537. 
14. COSMIC (Catalouge of somatic mutations in cancer) [database on the Internet]. Hinxton, UK: Wellcome Trust Sanger Institute. Available from: http:/cancer.sanger.ac.uk/cell_lines/browse/tissue?ss=NS\&sn=cervix. Accessed October 29, 2014.

15. Ojesina AI, Lichtenstein L, Freeman SS, et al. Landscape of genomic alterations in cervical carcinomas. Nature. 2014;506(7488): 371-375.

16. Schilder RJ, Sill MW, Lee YC, Mannel R. A Phase II trial of erlotinib in recurrent squamous cell carcinoma of the cervix: a Gynecologic Oncology Group study. Int J Gynecol Cancer. 2009;19(5):929-933.

17. Nogueira-Rodrigues A, do Carmo CC, Viegas C, et al. Phase I trial of erlotinib combined with cisplatin and radiotherapy for patients with locally advanced cervical squamous cell cancer. Clin Cancer Res. 2008;14(19):6324-6329.

18. Nogueira-Rodrigues A, Moralez G, Grazziotin R, et al. Phase II trial of erlotinib combined with cisplatin and radiotherapy in patients with locally advanced cervical cancer. Cancer. 2014;120(8):1187-1193.

19. Goncalves A, Fabbro M, Lhomme C, et al. A Phase II trial to evaluate gefitinib as second- or third-line treatment in patients with recurring locoregionally advanced or metastatic cervical cancer. Gynecol Oncol. 2008;108(1):42-46.

20. Sharma DN, Rath GK, Julka PK, Gandhi AK, Jagadesan P, Kumar S. Role of gefitinib in patients with recurrent or metastatic cervical carcinoma ineligible or refractory to systemic chemotherapy: first study from Asia. Int J Gynecol Cancer. 2013;23(4):705-709.

21. Hertlein L, Lenhard M, Kirschenhofer A, et al. Cetuximab monotherapy in advanced cervical cancer: a retrospective study with five patients. Arch Gynecol Obstet. 2011;283(1):109-113.

22. Santin AD, Sill MW, McMeekin DS, et al. Phase II trial of cetuximab in the treatment of persistent or recurrent squamous or non-squamous cell carcinoma of the cervix: a Gynecologic Oncology Group study. Gynecol Oncol. 2011;122(3):495-500.

23. Farley J, Sill MW, Birrer M, et al. Phase II study of cisplatin plus cetuximab in advanced, recurrent, and previously treated cancers of the cervix and evaluation of epidermal growth factor receptor immunohistochemical expression: a Gynecologic Oncology Group study. Gynecol Oncol. 2011;121(2):303-308.

24. Kurtz JE, Hardy-Bessard AC, Deslandres M, et al. Cetuximab, topotecan, and cisplatin for the treatment of advanced cervical cancer: a Phase II GINECO trial. Gynecol Oncol. 2009;113(1):16-20.

25. Moore KN, Sill MW, Miller DS, et al. A Phase I trial of tailored radiation therapy with concomitant cetuximab and cisplatin in the treatment of patients with cervical cancer: a Gynecologic Oncology Group study. Gynecol Oncol. 2012;127(3):456-461.

26. von Knebel Doeberitz M, Gissmann L, zur Haussen H. Growth-regulating functions of human papillomavirus early gene products in cervical cancer cells acting dominant over enhanced epidermal growth factor receptor expression. Cancer Res. 1990;50(12):3730-3736.

27. Candelaria M, Arias-Bonfill D, Chavez-Blanco A, et al. Lack in efficacy for imatinib mesylate as second-line treatment of recurrent or metastatic cervical cancer expressing platelet-derived growth factor receptor alpha. Int J Gynecol Cancer. 2009;19(9):1632-1637.

28. Mackay HJ, Tinker A, Winquist E, et al. A Phase II study of sunitinib in patients with locally advanced or metastatic cervical carcinoma: NCIC CTG Trial IND.184. Gynecol Oncol. 2010;116(2):163-167.

29. Tinker AV, Ellard S, Welch S, et al. Phase II study of temsirolimus (CCI-779) in women with recurrent, unresectable, locally advanced, or metastatic carcinoma of the cervix. A trial of the NCIC Clinical Trials Group (NCIC CTG IND 199). Gynecol Oncol. 2013;130(2):269-274.

30. Temkin SM, Yamada SD, Fleming GF. A Phase I study of weekly temsirolimus and topotecan in the treatment of advanced and/or recurrent gynecologic malignancies. Gynecol Oncol. 2010;117(3):473-476.

31. Gaffney DK, Winter K, Dicker AP, et al. Efficacy and patterns of failure for locally advanced cancer of the cervix treated with Celebrex (celecoxib) and chemoradiotherapy in RTOG 0128. Int J Radiat Oncol Biol Phys. 2007;69(1):111-117.
32. Tan S, de Vries EG, van der Zee AG, de Jong S. Anticancer drugs aimed at E6 and E7 activity in HPV-positive cervical cancer. Curr Cancer Drug Targets. 2012;12(2):170-184.

33. Ghosh U, Bhattacharyya NP. Induction of apoptosis by the inhibitors of poly(ADP-ribose) polymerase in HeLa cells. Mol Cell Biochem. 2009;320(1-2):15-23.

34. Reinbolt RE, Hays JL. The role of PARP inhibitors in the treatment of gynecologic malignancies. Front Oncol. 2013;3:237.

35. Duenas-Gonzalez A, Lizano M, Candelaria M, Cetina L, Arce C, Cervera E. Epigenetics of cervical cancer. An overview and therapeutic perspectives. Mol Cancer. 2005;4:38.

36. ManiE, MedinaLA, Isaac-Olive K, Duenas-GonzalezA. Radiosensitization of cervical cancer cells with epigenetic drugs hydralazine and valproate. Eur J Gynaecol Oncol. 2014;35(2):140-142.

37. Candelaria M, Cetina L, Perez-Cardenas E, et al. Epigenetic therapy and cisplatin chemoradiation in FIGO stage IIIB cervical cancer. Eur J Gynaecol Oncol. 2010;31(4):386-391.

38. Coronel J, Cetina L, Pacheco I, et al. A double-blind, placebo-controlled, randomized Phase III trial of chemotherapy plus epigenetic therapy with hydralazine valproate for advanced cervical cancer. Preliminary results. Med Oncol. 2011;28(Suppl 1):S540-S546.

39. Stafl A, Matinngly RF. Angiogenesis of cervical neoplasia. Am J Obstet Gynecol. 1975;121(6):845-852.

40. Guidi AJ, Abu-Jawdeh G, Berse B, et al. Vascular permeability factor (vascular endothelial growth factor) expression and angiogenesis in cervical neoplasia. J Natl Cancer Inst. 1995;87(16):1237-1245.

41. Lee JS, Kim HS, Park JT, Lee MC, Park CS. Expression of vascular endothelial growth factor in the progression of cervical neoplasia and its relation to angiogenesis and p53 status. Anal Quant Cytol Histol. 2003;25(6):303-311.

42. Loncaster JA, Cooper RA, Logue JP, Davidson SE, Hunter RD, West CM. Vascular endothelial growth factor (VEGF) expression is a prognostic factor for radiotherapy outcome in advanced carcinoma of the cervix. Br J Cancer. 2000;83(5):620-625.

43. Cheng WF, Chen CA, Lee CN, Wei LH, Hseih FJ, Hseih CY. Vascular endothelial growth factor and prognosis of cervical carcinoma. Obstet Gynecol. 2000;96(5 Pt 1):721-726.

44. Fujiwaki R, Hata K, Iida K, Maede Y, Miyazaki K. Vascular endothelial growth factor expression in progression of cervical cancer: correlation with thymidine phosphorylase expression, angiogenesis, tumor cell proliferation, and apoptosis. Anticancer Res. 2000;20(2B):1317-1322.

45. Wright JD, Viviano D, Powell MA, et al. Bevacizumab combination therapy in heavily pretreated, recurrent cervical cancer. Gynecol Oncol. 2006;103(2):489-493.

46. Tan SJ, Juan JH, Fu PT, Yu MH, Lai HC. Chemotherapy with low-dose bevacizumab and carboplatin in the treatment of a patient with recurrent cervical cancer. Eur J Gynaecol Oncol. 2010;31(3):350-353.

47. Monk BJ, Sill MW, Burger RA, Gray HJ, Buekers TE, Roman LD. Phase II trial of bevacizumab in the treatment of persistent or recurrent squamous cell carcinoma of the cervix: a Gynecology Oncology Group study. J Clin Oncol. 2009;27(7):1069-1074.

48. Zighelboim I, Wright JD, Gao F, et al. Multicenter Phase II trial of topotecan, cisplatin, and bevacizumab for recurrent or persistent cervical cancer. Gynecol Oncol. 2013;130(1):64-68.

49. Schefter T, Winter K, Kwon JS, et al. RTOG 0417: efficacy of bevacizumab in combination with definitive radiation therapy and cisplatin chemotherapy in untreated patients with locally advanced cervical carcinoma. Int J Radiat Oncol Biol Phys. 2014;88(1):101-105.

50. Duenas-Gonzalez A, Zarba JJ, Patel F, et al. Phase III, open-label, randomized study comparing concurrent gemcitabine plus cisplatin and radiation followed by adjuvant gemcitabine and cisplatin versus concurrent cisplatin and radiation in patients with stage IIB to IVA carcinoma of the cervix. J Clin Oncol. 2011;29(13):1678-1685.

51. Monk BJ, Mas Lopez L, Zarba JJ, et al. Phase II, open-label study of pazopanib or lapatinib monotherapy compared with pazopanib plus lapatinib combination therapy in patients with advanced and recurrent cervical cancer. J Clin Oncol. 2010;28(22):3562-3569. 
52. Monk BJ, Pandite LN. Survival data from a Phase II, open-label study of pazopanib or lapatinib monotherapy in patients with advanced and recurrent cervical cancer. J Clin Oncol. 2011;29(36):4845.

53. Wedge SR, Kendrew J, Hennequin LF, et al. AZD2171: a highly potent, orally bioavailable, vascular endothelial growth factor receptor-2 tyrosine kinase inhibitor for the treatment of cancer. Cancer Res. 2005;65(10):4389-4400

54. Diaz-Padilla I, Siu LL. Brivanib alanitate for cancer. Expert Opin Investig Drugs. 2011;20(4):577-586.

55. Wise-Draper TM, Wells SI. Papillomavirus E6 and E7 proteins and their cellular targets. Front Biosci. 2008;13:1003-1017.

56. Munger K, Scheffner M, Huibregtse JM, Howley PM. Interactions of HPV E6 and E7 oncoproteins with tumour suppressor gene products. Cancer Surv. 1992;12:197-217.

57. Narisawa-Saito M, Kiyono T. Basic mechanisms of high-risk human papillomavirus-induced carcinogenesis: roles of E6 and E7 proteins. Cancer Sci. 2007;98(10):1505-1511.

58. Yugawa T, Kiyono T. Molecular mechanisms of cervical carcinogenesis by high-risk human papillomaviruses: novel functions of E6 and E7 oncoproteins. Rev Med Virol. 2009;19(2):97-113.

59. Goodwin EC, DiMaio D. Repression of human papillomavirus oncogenes in HeLa cervical carcinoma cells causes the orderly reactivation of dormant tumor suppressor pathways. Proc Natl Acad Sci U S A. 2000;97(23):12513-12518.

60. Macville M, Schrock E, Padilla-Nash H, et al. Comprehensive and definitive molecular cytogenetic characterization of HeLa cells by spectral karyotyping. Cancer Res. 1999;59(1):141-150.

61. Sima N, Wang W, Kong D, et al. Interference against HPV16 E7 oncogene leads to viral E6 and E7 suppression in cervical cancer cells and apoptosis via upregulation of Rb and p53. Apoptosis. 2008;13(2): 273-281.

62. Jiang M, Milner J. Selective silencing of viral gene expression in HPVpositive human cervical carcinoma cells treated with siRNA, a primer of RNA interference. Oncogene. 2002;21(39):6041-6048.

63. Sima N, Wang S, Wang W, et al. Antisense targeting human papillomavirus type $16 \mathrm{E} 6$ and E7 genes contributes to apoptosis and senescence in SiHa cervical carcinoma cells. Gynecol Oncol. 2007; 106(2):299-304

64. Bousarghin L, Touze A, Gaud G, et al. Inhibition of cervical cancer cell growth by human papillomavirus virus-like particles packaged with human papillomavirus oncoprotein short hairpin RNAs. Mol Cancer Ther. 2009;8(2):357-365.

65. Gu W, Putral L, Hengst K, et al. Inhibition of cervical cancer cell growth in vitro and in vivo with lentiviral-vector delivered short hairpin RNA targeting human papillomavirus E6 and E7 oncogenes. Cancer Gene Ther. 2006;13(11):1023-1032.

66. Putral LN, Bywater MJ, Gu W, et al. RNA interference against human papillomavirus oncogenes in cervical cancer cells results in increased sensitivity to cisplatin. Mol Pharmacol. 2005;68(5):1311-1319.
67. Moon MS, Lee CJ, Um SJ, Park JS, Yang JM, Hwang ES. Effect of BPV1 E2-mediated inhibition of E6/E7 expression in HPV16-positive cervical carcinoma cells. Gynecol Oncol. 2001;80(2):168-175.

68. Lea JS, Sunaga N, Sato M, et al. Silencing of HPV 18 oncoproteins with RNA interference causes growth inhibition of cervical of cervical cancer cells. Reprod Sci. 2007;14(1):20-28.

69. Zhou Y, Zhang C, Liang W. Development of RNAi technology for targeted therapy - a track of siRNA based agents to RNAi therapeutics. J Control Release. Epub May 6, 2014.

70. Zhao CY, Szekely L, Bao W, Selivanova G. Rescue of $\mathrm{p} 53$ function by small-molecule RITA in cervical carcinoma by blocking E6-mediated degradation. Cancer Res. 2010;70(8):3372-3381.

71. Mungala R, Kausar H, Munjal C, Gupta RC. Withaferin A induces p53-dependent apoptosis by repression of HPV oncogenes and upregulation of tumor suppressor proteins in humancervical cancer cells. Carcinogenesis. 2011;32(11):1697-1705.

72. Allen KL, Tschantz DR, Awad KS, Lynch WP, DeLucia AL. A plant lignan, 3'-O-methyl-nordihydroguaiaretic acid, suppresses papillomavirus E6 protein function, stabilizes p53 protein, and induces apoptosis in cervical tumor cells. Mol Carcinog. 2007;46(7):564-575.

73. Beerheide W, Sim MM, Tan YJ, Bernard HU, Ting AE. Inactivation of the human papillomavirus-16 E6 oncoprotein by organic disulfides. Bioorg Med Chem. 2000;8(11):2549-2560.

74. Baleja JD, Cherry JJ, Liu Z, et al. Identification of inhibitors to papillomavirus type $16 \mathrm{E} 6$ protein based on three-dimensional structures of interacting proteins. Antiviral Res. 2006;72(1):49-59.

75. Cho Y, Cho C, Joung O, Lee K, Park S, Yoon D. Development of screening systems for drugs against human papillomavirus-associated cervical cancer: based on E6-E6AP binding. Antiviral Res. 2000;47(3): 199-206.

76. Abdulkarim B, Sabri S, Deutsch E, et al. Antiviral agent cidofovir restores p53 function and enhances the radiosensitivity in HPVassociated cancers. Oncogene. 2002;21(15):2334-2346.

77. Sirianni N, Wang J, Ferris RL. Antiviral activity of cidofovir on a naturally human papillomavirus-16 infected squamous cell carcinoma of the head and neck (SCCHN) cell line improves radiation sensitivity. Oral Oncol. 2005;41(4):423-428.

78. Yang Y, Zhao X, Chen W, et al. Effects of cidofovir on human papillomavirus-positive cervical cancer cells xenografts in nude mice. Oncol Res. 2010;18(11-12):519-527.

79. Snoeck R, Andrei G, De Clercq E. Cidofovir in the treatment of HPVassociated lesions. Verh K Acad Geneeskd Belg. 2001;63(2):93-120.

80. Van Pachterbeke C, Bucella D, Rozenberg S, et al. Topical treatment of CIN 2+ by cidofovir: results of a Phase II, double-blind, prospective, placebo-controlled study. Gynecol Oncol. 2009;115(1):69-74.
International Journal of Women's Health

\section{Publish your work in this journal}

The International Journal of Women's Health is an international, peerreviewed open-access journal publishing original research, reports, editorials, reviews and commentaries on all aspects of women's healthcare including gynecology, obstetrics, and breast cancer. The manuscript management system is completely online and includes

\section{Dovepress}

a very quick and fair peer-review system, which is all easy to use. Visit http://www.dovepress.com/testimonials.php to read real quotes from published authors. 